Classification

Physics Abstracts

$36.20 \mathrm{E}-46.60-78.35$

\title{
Observation of scaling in semi-dilute theta solutions using dynamic light scattering
}

\author{
A.-M. Hecht, H. B. Bohidar and E. Geissler \\ Laboratoire de Spectrométrie Physique (*), Université de Grenoble I, B.P. 68, \\ 38402 St Martin d'Hères Cedex, France
}

(Reçu le 4 novembre 1983, accepté le 7 décembre 1983)

\begin{abstract}
Résumé. - Nous présentons des mesures préliminaires du coefficient de diffusion collectif $D$ dans des solutions semi-diluées de polystyrène dans du cyclohexane à $35^{\circ} \mathrm{C}$ par diffusion quasi élastique de lumière. Les concentrations $c$ sont supérieures à la concentration critique d'enchevêtrement. Aux petits angles de diffusion, $D \propto c^{0,94 \pm 0,08}$; aux grands angles, $D$ donne le module de cisaillement de la solution. Les résultats étayent une théorie récente des solutions thêta due à $F$. Brochard.
\end{abstract}

\begin{abstract}
We present preliminary measurements of the collective diffusion coefficient $D$ of high molecular weight polystyrene in semi-dilute solution with cyclohexane at the theta temperature, using dynamic light scattering. The concentrations $c$ used were all well in excess of the critical entanglement concentration. For small scattering anglès, $D \propto c^{0.94 \pm 0.08}$. For large angles, $D$ gives the shear (plateau) modulus of the solution. The results provide strong support for a recent theory of theta solutions due to F. Brochard.
\end{abstract}

In semi-dilute polymer solutions the relaxation rate of concentration fluctuations is defined by a collective diffusion constant $D$. Scaling theory [1] has been shown to account satisfactorily for the behaviour of such solutions when excluded volume interactions are present. In theta solutions, however, where the polymer-polymer repulsion is compensated by an equal polymersolvent interaction (i.e. zero excluded volume), the experimental evidence is contradictory. According to the simplest scaling picture, polymer solutions with zero excluded volume obey Gaussian statistics, and $D$ should vary linearly with $c$, the concentration (measured in $\mathrm{g}$ of polymer per unit volume of solution). Experimental observations using dynamic light scattering [2-4] have indicated variations of the form $D \propto c^{\prime \prime}$, where the exponent $m$ lies in the range 0.2 to 0.7 . In contrast, macroscopic measurements using a classical gradient diffusion technique have given good agreement with the simple scaling prediction [5].

An explanation for this dichotomy has recently been proposed by Brochard [6]. For the length scale of interest $Q \xi<1$ (where $\mathbf{Q}$ is the transfer wave vector of the observation and $\xi$ is the correlation length of the fluctuations of the monomer concentration) there are two distinct elastic moduli in the solution, each having different properties. Entanglements between different polymer chains are assumed to form a transient network of lifetime $T_{\mathrm{R}}$; the shear modulus $G$

$\left(^{*}\right)$ CNRS associate laboratory. 
of this continuously changing network is understood to be proportional to the probability of two polymer strands coming into contact, i.e.

$$
G=G_{0} c^{2} .
$$

The osmotic modulus $K_{\mathrm{os}}$, meanwhile, is determined in theta solutions by three-body interactions. since the excluded volume is zero [7]. Thus

$$
K_{\mathrm{os}}=K_{\mathrm{os}}^{0} c^{3} \text {. }
$$

The collective diffusion coefficient is [8]

$$
D=\frac{K_{\mathrm{os}}+4 G+3}{f},
$$

where the friction coefficient $f$ in theta conditions is proportional [1] to $c^{2} . D$ may be obtained from the relaxation rate $\Gamma$ of the field correlation function of laser light scattered by the concentration fluctuations

$$
\Gamma=D Q^{2} \text {. }
$$

If $\Gamma T_{\mathrm{R}}<1$ then the transient network relaxes before it can contribute to the establishment of the equilibrium concentration, and hence $D \simeq K_{\mathrm{os}} / f$ : thus, for small $Q, D$ is proportional to $c$. In the opposite case, when $\Gamma T_{\mathrm{R}}>1$, since $K_{\mathrm{os}} \ll G$ for small $c$, it follows that $D$ should be independent of concentration.

In this letter we report observations using dynamic light scattering of the linear concentration dependence of $D$, as well as the first measurements of the storage modulus $G$ in theta solutions in the semi-dilute range. The observations described here all concern the region $Q \xi<1$ (the greatest value of $Q \xi$ being about 0.6 ); it follows that internal modes are not explored here.

The experiments were performed using atactic polystyrene of $M_{\mathrm{w}}=2.6 \times 10^{7}$ daltons, with polydispersity $M_{\mathrm{w}} / M_{\mathrm{n}}<1.3$, obtained through Interchim. France. For this preliminary experiment, three samples were prepared, using high purity cyclohexane as a solvent, with concentrations $0.0291,0.0567$ and $0.0954 \mathrm{~g} \mathrm{~cm}^{-3}$.

It is usually assumed [1] that the lower limit of the semi-dilute range is set by $c^{*}=M_{\mathrm{w}} / A r_{\mathrm{G}}^{3}$. where $A$ is Avogadro's number and $r_{\mathrm{G}}$ is the unperturbed radius of gyration of the polymer coil. In our case, $c^{*}$ is approximately $0.015 \mathrm{~g} \mathrm{~cm}^{-3}$, so that our samples lie within the semi-dilute region. However, $c^{*}$ is not the only relevant parameter : for viscoelastic observations, the crossover concentration is $c_{e}$, the concentration below with entanglements are absent. For semidilute solutions with excluded volume, $c_{\mathrm{e}} / c^{*} \gg 1$ [9]. The opposite is true in theta solutions. If $M_{\mathrm{e}}$ defines the mean mass of polymer chain between entanglements, then the shear modulus is given by [7]

$$
G=c k T A / M_{\mathrm{e}},
$$

which, together with (1) gives

$$
c=A k T / G_{0} M_{\mathrm{e}} .
$$

The critical entanglement concentration thus occurs when $M_{\mathrm{e}}=M_{\mathrm{w}}$ in (6). For the molecular weight used in this experiment, $c \simeq 5 \times 10^{-4} \mathrm{~g} \mathrm{~cm}^{-3}$, which is about two orders of magnitude smaller than $c^{*}$. The concentration range investigated here thus lies comfortably inside the entangled semi-dilute range.

The samples were prepared and sealed in rectangular glass fluorescence cells, the optical path of which was short $(2 \mathrm{~mm})$ in order to reduce multiple scattering. They were then kept in an oven at $40^{\circ}-45^{\circ} \mathrm{C}$ for more than two years before use, since it has been suggested that previous failure to observe the linear dependence of $D$ on $c$ was due to incomplete dissolution 
of the polystyrene in the theta solvent. A standard dynamic light scattering set-up was used [4], working in the heterodyne mode, with observations being made at forward angles, at $90^{\circ}$, and also in backscattering, using both the $632.8 \mathrm{~nm}$ line of a $15 \mathrm{~mW}$ He-Ne laser (SP 124) and the $514.5 \mathrm{~nm}$ line of an argon ion laser (SP 164). Heterodyning was ensured principally by the scattering from dust particles and imperfections in the solutions, and, in the forwards and backwards scattering geometries, also by flare from the glass-solution interface. In principle, the most unfavorable situation for meeting the heterodyne conditions occurs for $90^{\circ}$ scattering from the least concentrated solution; the measured ratio of the intensity of the reference signal to the signal from the concentration fluctuations in this case varied between 80 and $100:$ the signal is therefore fully heterodyned. The temperature of the samples was stabilized to $35.0 \pm 0.1{ }^{\circ} \mathrm{C}$.

The choice of the square cells precluded observations being made in a range of $Q$ between about $7 \times 10^{4}$ and $2 \times 10^{5} \mathrm{~cm}^{-1}$. The correlation spectra were analysed using a two-cumulant fit ; for scattering angles $\theta$ between $30^{\circ}$ and $40^{\circ}$, however, no unique fit could be found for the spectra, as the value of the first cumulant $\bar{\Gamma}$ depended on the correlator delay time. We conclude that the gap in the observed range of $Q$ overlies the transition region $\bar{\Gamma} T_{\mathrm{R}} \simeq 1$. To avoid this region, the averages of the low frequency diffusion coefficients were calculated using only the results from the two lowest scattering angles, namely $15^{\circ}$ and $20^{\circ}$. Figure 1 shows the measured values of $\bar{\Gamma} / Q^{2}$ as a function of $Q$ for the three samples.

Because of the finite volume fraction $\phi$ of the polymer, in order to obtain $D, \bar{\Gamma} / Q^{2}$ must be divided [10] by the factor $(1-\phi)$,

$$
D=\frac{\bar{\Gamma}}{Q^{2}(1-\phi)} \text {. }
$$

Figure 2 shows a double logarithmic representation of $D v s$. $c$, where in the lower curve $D$ is an average of the data from the two smallest scattering angles, and in the upper curve the average is taken over the three largest wave vectors. For the low $Q$ values, $D$ is given by

$$
\log D=-(5.64 \pm 0.10)+(0.94 \pm 0.08) \log c .
$$

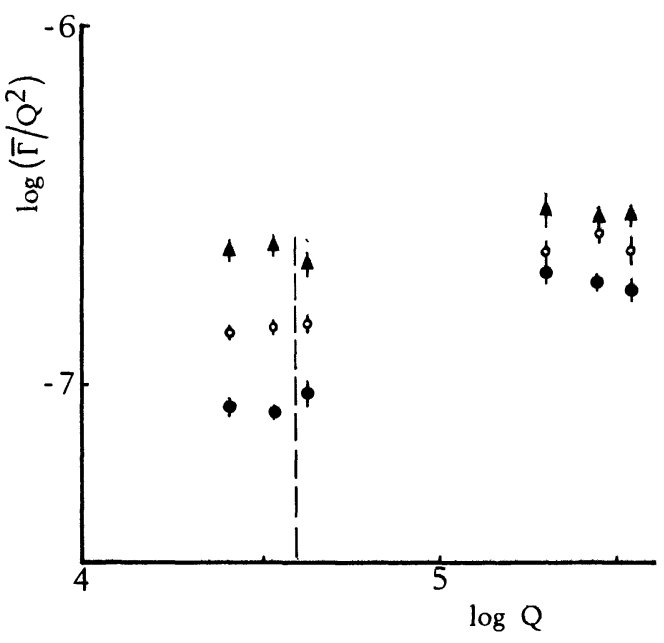

Fig. 1. - Log $\bar{\Gamma} / Q^{2}$ versus $\log Q$, where $\bar{\Gamma}\left(\mathrm{s}^{-1}\right)$ is the first cumulant of the dynamic light scattering correlation function and $Q$ the scattering wave vector $\left(\mathrm{cm}^{-1}\right)$. Filled circles $\bullet: c=0.0291 \mathrm{~g} \mathrm{~cm}^{-3}$; open circles $\bigcirc: c=0.0567 \mathrm{~g} \mathrm{~cm}^{-3}$; triangles $\Delta: c=0.0954 \mathrm{~g} \mathrm{~cm}^{-3}$. In the gap to the right of the vertical broken line the spectra do not admit of a simple decomposition. 


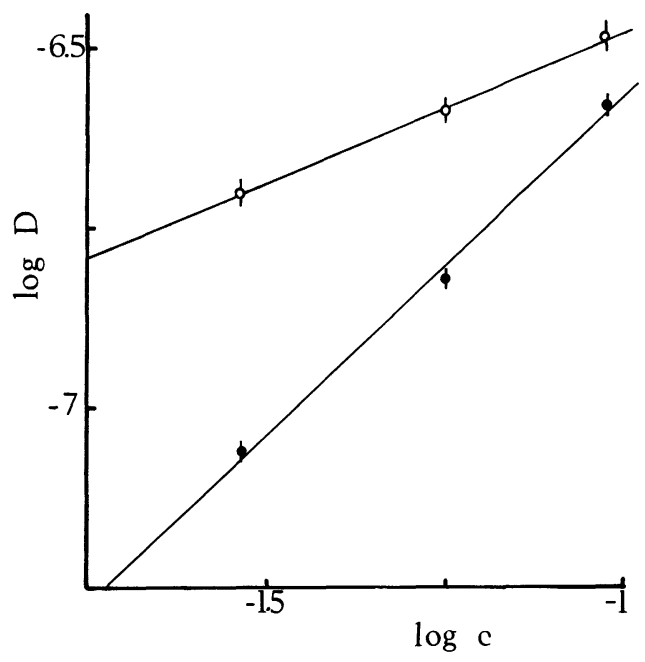

Fig. 2. - Plot of $\log D\left(\mathrm{~cm}^{2} \mathrm{~s}^{-1}\right)$ vs. $\log c\left(\mathrm{~g} \mathrm{~cm}^{-3}\right)$. Filled circles $\bullet$ average of the results from scattering angles $15^{\circ}$ and $20^{\circ}$; the slope of the straight line is $0.94 \pm 0.08$; open circles $O$ : average of the high $Q$ results; the slope of the straight line shown is $0.42 \pm 0.02$.

For the high $Q$ range, the average exponent is

$$
m=0.42 \pm 0.02 \text {. }
$$

The first of these curves is in agreement within experimental error with the predicted linear relation between $D$ and $c$. The second is an improper scaling relation whose exponent has no physical significance. Its correct representation can be found from (3) which becomes

$$
D=\frac{4}{3} \frac{G_{0}}{f_{0}}+\frac{K_{\mathrm{os}}^{0}}{f_{0}} c,
$$

where $f=f_{0} c^{2}$.

Figure 3 shows a linear plot of $D v s$. $c$ for the high $Q$ region. The best straight line through

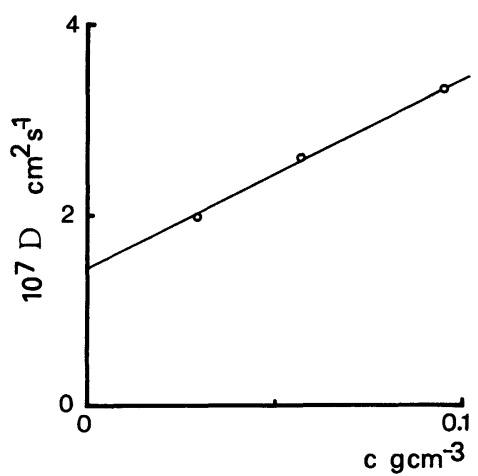

Fig. 3. - Linear representation of the high $Q$ values of $D$ shown in figure 2 . The intercept of the least square line at $c=0$ is $(1.45 \pm 0.07) \times 10^{-7} \mathrm{~cm}^{2} \mathrm{~s}^{-1}$. 
the three points is

$$
D=(1.45 \pm 0.07) \times 10^{-7}+(2.0 \pm 0.1) \times 10^{-6} c\left(\mathrm{~cm}^{2} \mathrm{~s}^{-1}\right) .
$$

According to the model [6], the coefficient of $c$, namely $K_{\mathrm{os}}^{0} / f_{0}$, appearing in (10) should be the same in the low $Q$ region as in the high $Q$ region. Equation (8) gives

$$
K_{\mathrm{os}}^{0} / f_{0}=(2.3 \pm 0.5) \times 10^{-6} \mathrm{~cm}^{-2} \mathrm{~s}^{-1}\left(\mathrm{~g} \mathrm{~cm}^{-3}\right)^{-1},
$$

which agrees within experimental error with the coefficient of $c$ in (11).

Now $K_{\mathrm{os}}^{0}$ has been determined independently for this system from measurements of the intensity of dynamically scattered light : from the low concentration results of reference [4] one gets

$$
K_{\mathrm{os}}^{0}=(5.3 \pm 0.6) \times 10^{7} \text { dynes } \mathrm{cm}^{-2}\left(\mathrm{~g} \mathrm{~cm}^{-3}\right)^{-3}
$$

By using the values of $G_{0} / f_{0}, K_{\text {os }}^{0} / f_{0}$ and $K_{\text {os }}^{0}$ contained in equations (11) through (13) respectively, one gets

$$
G_{0}=(2.5 \pm 0.7) \times 10^{6} \text { dynes } \mathrm{cm}^{-2}\left(\mathrm{~g} \mathrm{~cm}^{-3}\right)^{-2} .
$$

There is much experimental evidence [11] that in good solvents (1) holds right up to the concentration of the bulk polymer, $c=1 \mathrm{~g} \mathrm{~cm}^{-3}$, at which point $G=G_{0}$. A basic assumption of the Brochard model is that the same applies to theta solvents. If this assumption is valid, then the value for $G_{0}$ should correspond to the shear (plateau) modulus $G_{\mathrm{N}}^{0}$ in bulk polystyrene. The generally accepted value of $G_{\mathrm{N}}^{0}$ in the literature is [12] $2.0 \times 10^{6}$ dynes $\mathrm{cm}^{-2}$, which lies within the experimental error of the present measurements.

The preliminary results reported here indicate the validity of the Brochard model for semidilute theta solutions in the region $Q \xi<1$, in particular the existence of two elastic moduli with distinct concentration dependences. For polymer concentrations greater than about $1 \%$, these moduli are of comparable magnitude. This fact explains the observed residual concentration dependence of the collective diffusion coefficient at high relaxation rates. Moreover these observations permit a measurement of the shear modulus in semi-dilute theta solutions; to our knowledge, this has not hitherto been observed.

\section{Acknowledgments.}

One of us (HBB) acknowledges a Joliot-Curie Scholarship from the Commissariat à l'Energie Atomique. 


\section{References}

[1] De Gennes, P. G., Scaling Concepts in Polymer Physics (Cornell U.P.., Ithaca) 1978.

[2] Nose, T. and CHU, B., Macromolecules 12 (1979) 590.

[3] Lempert, W. and WANG, C. H., Molecular Phys. 42 (1981) 1027.

[4] Geissler, E. and Hecht, A.-M., J. Chem. Phys. 65 (1976) 103.

[5] Roots, J. and Nyström, B., Macromolecules 13 (1980) 1595.

[6] Brochard, F., J. Physique 44 (1983) 39.

[7] Flory, P. J., Principles of Polymer Chemistry (Cornell U.P. Ithaca) 1953.

[8] Tanaka, T., Hocker, L. O. and Benedek, G. B., J. Chem. Phys. 59 (1973) 5151.

[9] Geissler, E. and Hecht, A.-M., C.R. Hebd. Séan. Acad. Sci. 293 Série II (1981) 865.

[10] Geissler. E. and Hecht. A.-M.. J. Physique Lett. 40 (1979) L-173.

[11] Ferry, J. D., Viscoelustic Properties of Polymers (3rd ed. Wiley, New York) 1981.

[12] Plazek, D. J., Riande, E., Markowitz, H. and Ragupathi, N. J. Polymer Sci. Polymer Phys. 17 (1979) 2189. 